\title{
Parâmetros biológicos do estágio larval de Amblyomma cajennense (Fabricius, 1787) (Acari: Ixodidae) em coelhos
}

\section{Biological parameters of the larval stage of Amblyomma cajennense (Fabricius, 1787) (Acari: Ixodidae) in rabbits}

\author{
Márcia Cristina de Azevedo Prata ${ }^{1}$, Luciano da Silva Alonso ${ }^{1}$, Argemiro Sanavria²
}

\section{Resumo}

Objetivando maiores informações sobre as fases parasitária e não parasitária do Amblyomma cajennense, foram coletadas 473 fêmeas ingurgitadas provenientes de eqüinos. As fêmeas ingurgitadas pesavam, em média, $601,96 \pm 161,01 \mathrm{mg}$ e realizaram posturas entre 100 e 444 $\mathrm{mg}$, com média de $286,36 \pm 91,85 \mathrm{mg}$. A fase não parasitária foi avaliada sob condições controladas de laboratório (temperatura de $27^{\circ} \mathrm{C}$, umidade relativa do ar superior a $70 \%$ e $12 \mathrm{~h}$ de fotofase). Para cada $1 \mathrm{~g}$ de fêmeas ingurgitadas correponderam $507,88 \pm 88,61 \mathrm{mg}$ de postura. O período de pré-oviposição oscilou entre 4 e 7 dias, com média de $5,30 \pm 1,02$ dias; a incubação dos ovos foi realizada em média em 33,04 $\pm 1,69$ dias e o período de pré-eclosão foi de $37,94 \pm 1,48$ dias. A fase parasitária foi acompanhada a partir de infestações experimentais em coelhos, utilizando-se larvas de 3 a 18 dias de idade, provenientes das posturas das fêmeas coletadas. 0 ingurgitamento larval foi realizado entre 4 e 6 dias, com média de 5 dias. O período compreendido entre o desprendimento da metalarva e o término da ecdise larva/ninfa foi de 11,29 $\pm 1,19$ dias e não foi afetado nem pelo sexo nem pela cor da pelagem do coelho utilizado para ingurgitamento larval. Do total de metalarvas obtidas, 95\% completaram a ecdise.

Palavras chave: biologia; larvas; Amblyomma cajennense; Ixodidae

\section{Introdução}

Amplamente distribuído no continente americano, desde o sul do Texas nos Estados Unidos até a América do Sul, o Amblyomma cajennense (Fabricius, 1787) ou "carrapato estrela" é um parasita que ataca o homem e inúmeras espécies animais (Cooley e Kohls, 1944; Hoffmann, 1962). No Brasil, ocorre durante todos os meses do ano, sendo a forma adulta mais freqüente entre setembro e dezembro (Serra Freire, 1982). Parasita principalmente eqüinos e localiza-se preferencialmente na região perianal, nas axilas e no abdômen. Porém, em altas infestações, pode ser encontrado no interior do pavilhão auriculare em outras partes do corpo dos animais (Flechtmann, 1977).

Por ser um carrapato que realiza mudas fora do corpo do hospedeiro, necessitando três hospedeiros para completar seu ciclo, apresenta maior possibilidade de se infectar e transmitir patógenos. É considerado transmissor da babesiose (Horta e Figueiredo, 1914), tifo exantemático (Monteiro et al., 1931), piroplasmose eqüina (Flechtmann, 1977) e ehrlichiose (Massard, 1984). Acarreta alterações hematológicas, cutâneas e nervosas nos animais parasitados (Serra Freire, 1979), sendo incriminado como agente indutor de paralisia em ruminantes (Serra Freire, 1983).

Apesar das perdas com a produtividade e a saúde dos animais, poucos estudos foram realizados sobre os aspectos biológicos deste ixodídeo. Rohr (1909) e Travassos e Vallejo-Freire (1944) estudaram seu ciclo biológico. Drummond e Whetstone (1975) pesquisaram sua biologia sob condições controladas. Cunha (1978) avaliou o poder toxicóforo dos diferentes estágios parasitários sobre coeIhos. Olivieri e Serra Freire (1984), utilizando neolarvas $F_{1}$ provenientes de fêmeas ingurgitadas coletadas de bovinos, forneceram detalhes sobre a fase larval desse parasito em infestações experimentais em coelhos.

O presente trabalho foi realizado visando a obtenção de maiores informações sobre as fases parasitária e não parasitária do ciclo biológico do $A$. cajennense sob condições controladas, em laboratório, na tentativa de auxiliar o estabelecimento de estratégias de controle químico ou biológico deste ixodídeo.

\section{Material e Métodos}

A pesquisa foi realizada no Laboratório de Doenças Parasitárias do Departamento de Epidemiologia e Saúde Pública, no Instituto de Veterinária da Universidade Federal Rural do Rio de Janeiro, situada no município de

\footnotetext{
'Acadêmicos de Medicina Veterinária da UFRRJ, bolsistas de Iniciação Científica do CNPq

2 Departamento de Epidemiologia e Saúde Pública, Instituto de Veterinária, Universidade Federal Rural do Rio de Janeiro, UFFRJ, km 47 da Antiga Rodovia Rio-São Paulo, 23851-970 Seropédica, RJ, Brasil
} 
Seropédica, RJ, no período de março a setembro de 1994. Foram utilizadas 473 fêmeas ingurgitadas provenientes de eqüinos naturalmente infestados, criados extensivamente no município.

Após a coleta, as fêmeas ingurgitadas transferidas para o laboratório, foram limpas, pesadas em balança eletrônica com precisão de $0,001 \mathrm{~g}$, acondicionadas em grupos, de no máximo 10 , em placas de Petri e mantidas em estufa biológica para $\mathrm{BOD}$ a $27^{\circ} \mathrm{C}$, com umidade relativa superior a $70 \%$ e $12 \mathrm{~h}$ de fotofase. Vinte dias após o início da postura, os ovos foram pesados e acondicionados em seringas plásticas, contendo $0,100 \mathrm{~g}$ de ovos cada, mantidas em BOD, sob as condições descritas anteriormente, para a eclosão dos ovos.

Para acompanhamento da fase parasitária, foram realizadas 34 infestações em 31 coelhos adultos, de ambos os sexos, com pelagem uniforme nas cores branca ou preta. Cada coelho foi infestado com 3.200 a 4.800 larvas (entre 13 e 18 dias de idade) depositadas no pavilhão auricular, onde havia sido adaptado um capuz de pano aderido à pele, pela extremidade inferior, com pasta UNA (água destilada, gelatina em folha, glicerina e óxido de zinco). A extremidade superior do capuz era vedada com velcro, técnica esta modificada de Neitz et al. (1971). Os capuzes eram abertos diariamente para acompanhamento dos processos de fixação, ingurgitamento e desprendimento larval, até a queda de todas as metalarvas. Cinco dias após cada infestação, era efetuado o recolhimento das metalarvas, que eram contadas, acondicionadas em seringas e mantidas em BOD para efetuar a ecdise. Foi realizada a análise de variância (ANOVA) ao nível de significância de $5 \%$, para se verificar a existência de diferenças significativas quanto ao período de ecdise larva/ninfa em relação ao sexo e/ou à cor da pelagem do coelho utilizado para ingurgitamento larval.

\section{Resultados e Discussão}

O peso médio da fêmea ingurgitada foi de $601,96 \pm$ $161,01 \mathrm{mg}$, variando em intervalo de 340 a $1.220 \mathrm{mg}$. As fêmeas realizaram posturas com peso entre 100 e $444 \mathrm{mg}$ com média de $286,36 \pm 91,85 \mathrm{mg}$. O peso médio de postura por grama de fêmeas ingurgitadas foi de 507,88 $\pm 88,61$ $\mathrm{mg}$, oscilando entre 333,75 e $673,05 \mathrm{mg}$ (Tabela 1 ). 0 percentual de eclosão de ovos oscilou em torno de $95 \%$, o que pode ser explicado pelo fato de serem provenientes de fêmeas ingurgitadas coletadas de animais não tratados com carrapaticidas e mantidas em laboratório sob condições controladas.

Drummond e Whetstone (1975), analisando parâmetros biológicos de fêmeas ingurgitadas de $A$. cajennense coletadas de bovinos, registraram peso médio de $639 \mathrm{mg}$ para cada fêmea, variando entre 457 e $831 \mathrm{mg}$ e concluíram que, para cada $1 \mathrm{~g}$ de fêmeas, corresponde uma média de $618 \mathrm{mg}$ de postura (314 a $717 \mathrm{mg}$ ). A diferença entre os valores obtidos por esses autores e os do presente trabalho talvez tenha sido determinada pela espécie de hospedeiro do qual foram coletados os carrapatos. Daemon (1985), ao analisar parâmetros biológicos de Anocentor nitens, em condições de laboratório, a partir de fêmeas ingurgitadas coletadas de eqüinos e de bovinos, obteve valores de peso médio de cada fêmea ingurgitada e peso de postura por grama de fêmeas, mais elevados para a cepa proveniente de bovinos.

O período de ingurgitamento larval oscilou entre 4 e 6 dias, com uma média de 5 dias. Flechtmann (1977) obteve período de 3 a 6 dias, mas não informou sob que condições esses dados foram obtidos. Hooker et al. (1912) relataram oscilação entre 3 e 7 dias, com maior quantidade de larvas completando seu ingurgitamento entre 4 e 5 dias. Olivieri e Serra Freire (1984), realizando infestações experimentais em coelhos com larvas provenientes de fêmeas ingurgitadas coletadas de bovinos, verificaram um intervalo de 3 a 8 dias para o ingurgitamento larval.

Os períodos referentes à fase não parasitária (Tabela 2), por terem sido obtidos sob condições controladas de temperatura, umidade relativa do ar e fotoperíodo, foram bem mais curtos que os observados sob condições naturais. O período de pré-oviposição variou entre 4 e 7 dias, com uma média de $5,30 \pm 1,02$ dias. A incubação dos ovos foi efetuada em $33,04 \pm 1,69$ dias, em média, e o período de pré-eclosão (compreendido entre a queda da fêmea ingurgitada e o início da eclosão dos ovos por ela postos) foi de $37,94 \pm 1,48$ dias. O período compreendido entre o desprendimento da metalarva e o término da ecdise larva/ ninfa foi de 11,29 $\pm 1,19$ dias, oscilando entre 9 e 14 dias. $\mathrm{Na}$ literatura são registrados valores bem superiores aos do presente trabalho. Segundo Flechtmann (1977), o período de pré-oviposição oscila entre 11 e 12 dias; as posturas permanecem em incubação por 30 a 70 dias e a ecdise larva/ninfa leva de 18 a 26 dias para se completar. Olivieri e Serra Freire (1984), registraram período de 16,48 dias para o término da ecdise larva/ninfa, sob condições semelhantes às descritas no presente trabalho.

Do total de metalarvas obtidas, $95 \%$ completaram a ecdise. Olivieri e Serra Freire (1984) obtiveram 84,79\% de neoninfas para o total de metalarvas coletadas. Os valores referentes à ecdise relacionada ao sexo e à cor da pelagem dos coelhos em infestações experimentais com larvas e ninfas são apresentados na Tabela 3. A análise de variância ao nível de significância de $5 \%$ indicou que não há diferenças significativas quanto ao período de ecdise larva/ninfa relacionado ao sexo nem à cor da pelagem do hospedeiro, ou seja, o período de ecdise larva/ninfa não é afetado nem pelo sexo nem pela cor da pelagem do hospedeiro utilizado para ingurgitamento, quando são feitas infestações experimentais em coelhos. 
Tabela 1 - Peso médio da fêmea ingurgitada e peso das posturas por fêmea e por grama de fêmeas ingurgitadas, acompanhados dos respectivos desvio padrão (DP), coeficiente de variação (CV), limites inferior e superior e amplitude de variação para Amblyomma cajennense, sob condições controladas

\begin{tabular}{lcrrrc}
\hline Parâmetros biológicos & Média & DP & CV & $\begin{array}{c}\text { Limites } \\
\text { (inferior-superior) }\end{array}$ & $\begin{array}{c}\text { Amplitude variação } \\
\text { de }\end{array}$ \\
\hline Peso da fêmea ingurgitada $(\mathrm{mg})$ & 601,96 & 161,01 & 26,75 & $(340,00-220,00)$ & 880,00 \\
Peso da postura de cada fêmea ingurgitada $(\mathrm{mg})$ & 286,36 & 91,85 & 32,08 & $(100,00-444,00)$ & 344,00 \\
Peso da postura $(\mathrm{mg}) / \mathrm{g}$ de fêmeas ingurgitadas & 507,88 & 88,61 & 17,45 & $(333,75-673,05)$ & 339,30 \\
\hline
\end{tabular}

Tabela 2 - Valores referentes à fase não parasitária de Amblyomma cajennense, sob condições controladas

\begin{tabular}{lccccc}
\hline Parâmetros biológicos & Média & DP & CV & $\begin{array}{c}\text { Limites } \\
\text { (inferior-superior) }\end{array}$ & $\begin{array}{c}\text { Amplitude de } \\
\text { variação }\end{array}$ \\
\hline Período de pré-oviposição (dias) & 5,30 & 1,02 & 19,24 & $(4-7)$ & 3 \\
Período de incubação (dias) & 33,04 & 1,69 & 5,12 & $(29-37)$ & 8 \\
Período de pré-eclosão (dias) & 37,94 & 1,48 & 3,90 & $(36-42)$ & 6 \\
Período compreendido entre & & & & & \\
o desprendimento da metalarva & & & & $(9-14)$ & 5 \\
e o término da ecdise larva/ninfa (dias) & 11,29 & 1,19 & 10,54 & & \\
\hline
\end{tabular}

$\mathrm{DP}=$ desvio padrão; $\mathrm{CV}=$ coeficiente de variação

Tabela 3 - Valores da ecdise relacionada ao sexo e à cor da pelagem de coelhos utilizados para ingurgitamento, em infestações experimentais com ninfas de Amblyomma cajennense

\begin{tabular}{lcccc}
\hline $\begin{array}{l}\text { Coelho (sexo e } \\
\text { cor da pelagem) }\end{array}$ & $\begin{array}{c}\text { № de } \\
\text { infestações }\end{array}$ & $\begin{array}{c}\text { № de } \\
\text { coelhos }\end{array}$ & $\begin{array}{c}\text { Idade da } \\
\text { larva } \\
\text { infestante (dias) }\end{array}$ & $\begin{array}{c}\text { Período entre desprendimento } \\
\text { da metaninfa e término da } \\
\text { ecdise (dias) } \bar{X} \pm \text { DP } \\
\text { Limites (inferior - superior) }\end{array}$ \\
\hline Macho branco & 8 & 8 & 3 a 13 & $11,38 \pm 0,74(10-12)$ \\
Macho preto & 8 & 7 & 5 a 14 & $11,25 \pm 1,16(9-13)$ \\
Fêmea branca & 15 & 13 & 3 a 18 & $11,07 \pm 1,33(9-13)$ \\
Fêmea preta & 3 & 3 & 3 a 8 & $12,33 \pm 1,53(11-14)$ \\
\hline
\end{tabular}

$\bar{X}=$ média; $D P=$ desvio padrão

\section{Abstract \\ Biological parameters of the larval stage of Ambly- omma cajennense (Fabricius, 1787) (Acari: Ixodidae) in rabbits}

In an attempt to obtain further information on both parasitic and non-parasitic phases of Amblyomma cajennense, 473 engorged females from horses were collected. Females weighed $601.96 \pm 161.01 \mathrm{mg}$ on the average and made oviposition between 100 and $444 \mathrm{mg}$, with an average of $286.36 \pm 91.85 \mathrm{mg}$. Non-parasitic phase was assessed under laboratory conditions $\left(27^{\circ} \mathrm{C}\right.$ temperature, relative air humidity above $70 \%$ and $12 \mathrm{hr}$ photophase exposition). For $1 \mathrm{~g}$ females there was a correspondence of $507.88 \pm 88.61 \mathrm{mg}$ oviposition. Pre- oviposition period ranged from 4 to 7 days, with an average of $5.30 \pm 1.02$ days; egg incubation was made in $33.04 \pm$ 1.69 days on the average, and the pre-eclosio period was $37.94 \pm 1.48$ days. Parasitic phase was followed on the basis of experimental infestations in rabbits, using 3 to 18 day larvae from the oviposition of collected females. Larval ingurgitation was observed between 4 and 6 days with an average of 5 days. The period extending between loosening of metalarva and completion of larva/nymph ecdisis was $11.29 \pm 1.19$. This period was not affected by sex or hair color of rabbit used for larvae ingurgitation. Out of the total of metalarvae obtained, $95 \%$ completed ecdisis.

Key words: biology; larvae; Amblyomma cajennense; Ixodidae 


\section{Referências bibliográficas}

Cooley RA, Kohls GM 1944. The genus Amblyomma (Ixodidae) in the United States. J Parasitol 30:77-111.

Cunha DW da 1978. Estudos da toxicidade de alguns carrapatos comumente encontrados no Brasil (Acarina: Ixodidae). Dissertação (Mestrado), Universidade Federal Rural do Rio de Janeiro, Itaguaí, 89 pp.

Daemon E 1985. Biologia da fase não parasitária de Anocentor nitens (Neumann, 1897) (Acarina: Ixodidae) em condições de laboratório. Dissertação (Mestrado), Universidade Federal Rural do Rio de Janeiro, Itaguaí, 91 pp.

Drummond RO, Whetstone TM 1975. Oviposition of the cayenne tick, Amblyomma cajennense (F.) in the laboratory. Ann Entom Soc Am 68(2): 214-216.

Flechtmann CHW 1977. Ácaros de Importância Médico Veterinária. $2^{\mathrm{a}}$ ed., Liv. Nobel, São Paulo, 192 pp.

Hoffmann A 1962. Monographia de los Ixodoidea de Mexico, 1 Parte. Rev Soc Mex Hist Nat 23: 191-301.

Hooker WA, Bishopp FC, Wood HP 1912. The Life History and Bionomics of Some North American Ticks. U.S. Dept. Agricult., Bur. Entomol. Plant. Quar. Bull. n. 106, Washington, D.C., 239 pp.

Horta PP, Figueiredo AS 1914. Nutaliose dos equideos em Minas Gerais ("A mijadeira dos Poldrinhos"). Rev Vet Zootec Rio de Janeiro, 4(1): 3.

Massard C de A 1984. Ehrlichia bovis (Donatien \& Lestoquard, 1936): Diagnóstico, cultivo "in vitro" e aspectos epidemiológicos em bovinos no Brasil. Tese (Doutorado),
Universidade Federal Rural do Rio de Janeiro, Itaguaí, 113 pp.

Monteiro JL, Fonseca F, Prado A 1931. Pesquisas epidemiológicas sobre o Thypo Exantemático de São Paulo. Mem Inst Butantã 6: 139-173.

Neitz WO, Boughton F, Walters HS 1971. Laboratory investigation on the life cicle of the karoo paralysis tick (Ixodes rubicundus Neumann, 1904). Onderstepoort J Vet Res 38: 215-224.

Olivieri JA, Serra Freire NM 1984. Estádio larval do ciclo biológico de Amblyomma cajennense. Arq Univ Fed Rur Rio de Janeiro 7(2): 139-147.

Rohr CJ 1909. Estudos sobre Ixodidae do Brasil. Instituto Oswaldo Cruz, Rio de Janeiro, 200 pp.

Serra Freire NM 1979. Toxicidade de Amblyomma cajennense para ruminantes domésticos e sua significação como agente de uma nova forma de "tick paralysis". Tese (Doutorado), Universidade Federal Rural do Rio de Janeiro, Itaguaí, 119 pp.

Serra Freire NM 1982. Epidemiologia de Amblyomma cajennense: Ocorrência estacional e comportamento dos estádios não parasitários em pastagens do Estado do Rio de Janeiro. Arq Univ Fed Rur Rio de Janeiro 5(2): 187193.

Serra Freire NM 1983. Tick paralysis in Brazil. Trop Anim Hith Prod 15: 124-126.

Travassos J, Vallejo Freire A 1944. Criação artificial de Amblyomma cajennense para o preparo de vacina contra a febre maculosa. Mem Inst Butantã 18: 145-235. 